\title{
A MODEL FOR EVALUATING THE IMPACT OF AGGREGATED ROUTING INFORMATION ON NET- WORK PERFORMANCE
}

\author{
J.L.Rougier ${ }^{1 *}$, A.R.P.Ragozini' ${ }^{2}$, A. Gravey ${ }^{3}$, D. Kofman ${ }^{1}$ \\ ${ }^{1}$ Ecole Nationale Supérieure des Télécommunications. France. \\ ${ }^{2}$ Università Federico II di Napoli, Dipartimento di Ingegneria Elettronica e delle Telecomuni- \\ cazioni. Italy. \\ ${ }^{3}$ France Telecom - CNET. France \\ Corresponding Authors: rougier@email.enst.fr, ragozini@unina.it
}

\begin{abstract}
This paper presents a mathematical model for evaluating the impact of information aggregation on the performance of PNNI-driven ATM networks. The routing aggregation scheme affects both the performance and scalability of these networks. However, to date, little is still known on how the choice of PNNI configuration parameters influences the network behavior. A generic model for the aggregation process is proposed in order to study its impact on the network utilization and routing overhead. Random geometric considerations are used to obtain closed-form approximations of various performance measures.
\end{abstract}

Keywords: QoS Routing, Hierarchy, aggregation, PNNI, Performance Analysis, Random Geometry.

\section{INTRODUCTION}

\subsection{MOTIVATIONS}

In this paper, we analyze the trade-off between performance and scalability in PNNI-driven ATM networks. The PNNI protocol, defined by the ATMForum, provides dynamic, hierarchical and $Q o S$-sensitive routing capabilities. Unfortunately, the design and dimensioning of networks running PNNI is still not well known. For instance, the performance and scalability of PNNI are both affected by the routing aggregation scheme. However, very little is still known 
on how this scheme impacts the network performance (e.g., call blocking rate) or scalability (i.e., routing overhead).

\subsection{PNNI BASIS}

QoS routing. The PNNI standard [1] defines a routing protocol adapted for the specific characteristics of ATM networks. PNNI is a QoS-sensitive routing protocol, which means that the choice of the paths is based on the knowledge of network state and connection QoS requirements. QoS routing potentially increases the likelihood of accommodating calls with diverse QoS requirements while optimizing the network usage.

However, dynamic QoS routing requires that the switches exchange link attributes or metrics, such as link load information, which can consume considerable network resources. The routing computation based on this information can also become very expensive in terms of processing and memory consumption $[6 ; 9 ; 17]$. This means that the routing information (such as link loads) should only be exchanged on a regular basis (with a small frequency) or after significant events. However, the routing information updating policy is critical to network performances; a careful trade-off between accuracy and complexity must be found. In this paper, we shall neglect the discrepancies caused by the routing update scheme - thus assuming that all the nodes have up-todate routing information. The impact of stale routing information on network performance has been studied in a companion paper [15].

Hierarchy. The link information exchange, and the processing that it induces, can become prohibitive as the network size grows. PNNI ensures scalability by dividing the network into routing domains, called Peer-Groups - as depicted in Figure 1(a). A node in a Peer-Group (PG) has a complete view of the topology and routing information of the PG but only an aggregated view of the topology and routing information of the rest of the network. As information exchanged between different Peer-Groups is aggregated (i.e., summarized), the routing overhead is reduced. The impact of hierarchy on the routing overheads (e.g. routing table size, routing computation complexity) has been studied in $[10 ; 12 ; 14]$. In this paper, we analyze the impact of this hierarchical organization on network utilization.

Aggretation. The aggregation scheme aims at representing a whole peergroup, which potentially contains many links and nodes, with a small number of parameters. For instance, if the simple node representation [1] is used, the whole peer-group is seen as a single node ${ }^{1}$. In this case, the available bandwidth announced represents how much residual bandwidth could be expected by calls which have to cross the Peer-Group. A more precise alternative, is to use the complex node representation [1], where a peer-group is represented by an 


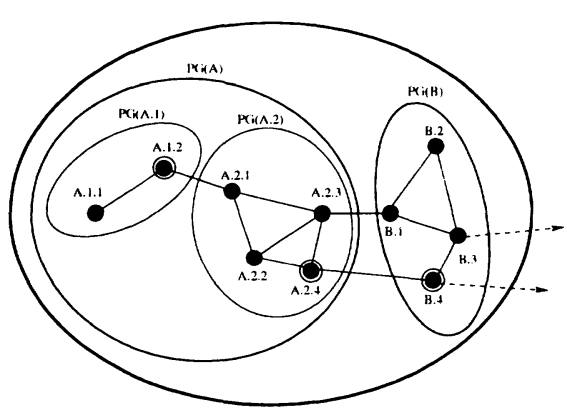

(a) Example of hierarchical clustering.

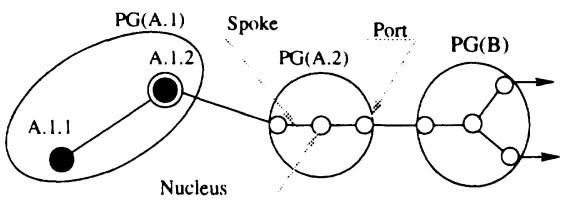

(b) Complex Node Representation

Figure 1 Hierarchy in PNNI

asymmetric star - thus announcing one set of link attributes and metrics for each entry point to the PG (see Figure 1(b)). The design of efficient and scalable aggregation schemes is difficult $[7 ; 8 ; 11]$, as it requires a delicate trade-off between scalability and routing accuracy (network optimization).

Source-Routing. PNNI is a source-routing protocol, which means that the path is chosen once at the source switch ${ }^{2}$. As a specific routing computation could not possibly be run at each call arrival, a set of precomputed paths for any possible destination is usually stored in each switch $[1 ; 8 ; 17]$. The source selects one of these available paths which fits the call QoS requirements using GCAC (Generic Call Admission Control). If none of the precomputed paths is feasible, then an on-demand routing computation may be used or the call can be rejected.

In a hierarchical network, some topological information is hidden by the aggregation scheme, making it impossible for the source to determine a complete path. However, a logical path through the Peer Groups is computed by the first switch based on aggregated routing information. The route is then refined at each entrance in a new Peer-Group in order to determine how to cross the selected domain.

Crankback. When selecting a path, a node uses the currently available information about resources and connectivity. That information may be inaccurate because of hierarchical aggregation for instance. Therefore, a call may be blocked along its specified route. A crankback procedure is then used for partially releasing the reserved resources. An alternate path over which to route the call may then be chosen. 


\subsection{FOCUS OF THIS WORK}

PNNI offers network designers a considerable amount of latitude in limiting routing overhead - at the expense of performances. In particular, both routing information aggregation and distribution can be controlled. Some recent studies, based on simulations, have analyzed the tradeoff between scalability and performance in PNNI $[7 ; 16 ; 17]$. Unfortunately, most studies analyzes on the routing update policy and do not consider the aggregation scheme. To the authors knowledge, no analytical results are available today.

In this paper, an analytical model is provided which allows to study the impact of aggregation on network performance (e.g. call blocking probability). Our results are based on the random-geometric model proposed in the next section. In section 3 various performance measures are expressed for a network with two hierarchical levels. Some results are analyzed in section 4. Conclusions and future work are presented in section 5. Technical considerations have been placed in appendices for the sake of clarity.

\section{THE MODEL}

In this paper, a random-geometric representation is used to model the network. Random geometry [5] has been recently applied with success to the performance evaluation of various networking problems [2; 14].

Physical Topology. The topology of the network is generated by stationary Poisson processes in the plane. At the physical level, the switches are represented by points of a Poisson process $\pi_{0}$ of intensity $\lambda_{0}$. In order to get a finite network, only the points which lie in a finite area $W$ will be considered. Without loss of generality, we shall assume that $W$ is a disc of unitary surface $(|W|=1)$, so that the average number of switches in the network is $\lambda_{0}$.

The connectivity between these nodes will be constructed by the so-called Delaunay Graph (see appendix A.1 ). A weighted graph is obtained by marking each link $i$ with its available bandwidth $X_{i}$ - other metrics or attributes such as the delays or jitter will not be considered. In order to limit the routing overhead, the values $\left\{X_{i}\right\}$ are normally broadcasted periodically or when a significant event occurs, so that the other nodes should only be able to observe estimations of the real bandwidths. In this paper, we shall concentrate on hierarchy and aggregation, and we shall neglect this phenomenon. The impact of stale routing information on network performances has been studied in a companion paper [15].

Hierarchy. In this paper, we shall consider a network wth two levels of hierarchy. A new stationary Poisson process $\pi_{1}$, of intensity $\lambda_{1}$, is introduced to divide the network into peer-groups. More precisely, the network is clustered 
using Voronoi tessellations [2; 5] (see appendix A.1 ) built with this new process. For each $u \in \pi_{1}$ we define a Peer-Group as a set containing all the nodes of $\pi_{0}$ which fall in the Voronoi cell $C_{u}\left(\pi_{1}\right)$.

The nodes at the higher level ( the "logical nodes") correspond to the points of $\pi_{1}$. The logical connectivity between nodes at the higher level is also modeled by a Delaunay Graph. Such a model is depicted in Figure 2(a).

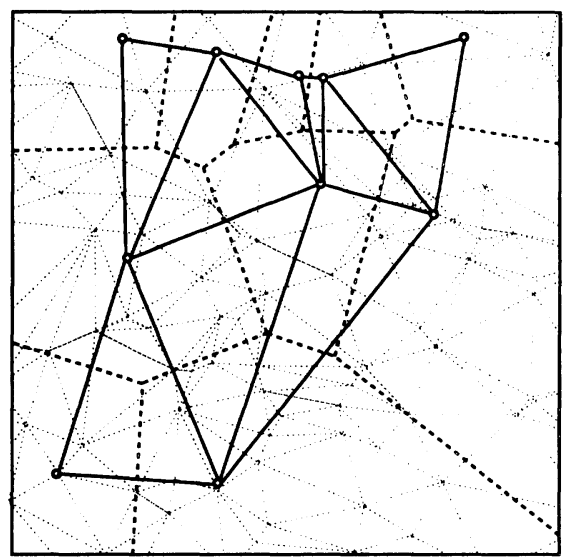

(a) Connectivity

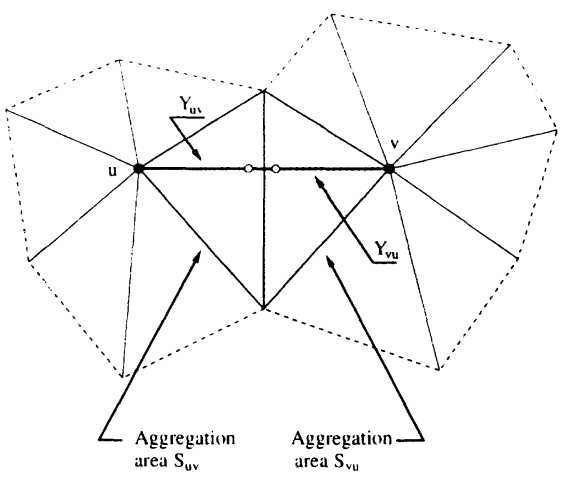

(b) Complex Node Represenation

Figure 2 The hierarchical model.

Aggregation model.. In this paper, a complex node representation as defined in [1] is used ${ }^{3}$. The nuclei will be chosen as the centroids of the Voronoi cells (see Figure 2(b)). The links joining each port to the nucleus are marked by the aggregated bandwidth that one could expect when trying to reach nodes at the "center" of the peer-group.

Aggregation strategies are not specified in the standards. In the following we propose a generic aggregation model ${ }^{4}$, as a function of its "aggressiveness". The cell is divided into non-overlapping areas (one for each port) as shown in Figure 2(b). The different aggregated values $Y_{u, \text {, }}$ are then computed independentely in each area: Given $\beta \in\left[0,1\left[\right.\right.$, the aggregated value $Y_{u, v}$ will be chosen as the $\beta$-sample quantile ${ }^{5}$ of the set $\left\{X_{i}\right\}$ in the corresponding area. $\beta$ can be seen as the "aggressiveness" of the aggregation strategy: For $\beta=0, Y_{u, v}$ is the minumum of all the available bandwidths, for $\beta \rightarrow 1$, the aggregated value is very optimistic as it corresponds to the maximum bandwidth available in the set. 
A logical link of level 1 , say $u v$, will then be marked by a single quantity $X_{u, v}^{1}=\min \left\{Y_{u, v}, Y_{v, u}\right\}$. We shall assume that the $\{Y$. $\}$ are independent and identically distributed ${ }^{6}$. This assumption implies that $\left\{X_{.,}^{1}\right\}$ is a set of independent and identically distributed random variables. Finally, we can introduce $\mathbf{p}_{1}=\mathbb{P}\left(X^{1}>b\right)$ and $\mathbf{q}_{1}=\mathbb{P}(Y>b)$ (omitting the link indications from the i.i.d. assumption) where $b$ is the amount of bandwidth ${ }^{7}$ requested by the used. It should be noted that, by definition, $\mathbf{p}_{1}=\mathbf{q}_{1}^{2}$.

Routing algorithm. We shall assume that the routing algorithm at the source switch is recursive (As discussed in [13]). Furthermore, we shall use precomputed paths. At each call arrival, a list of precomputed paths for the destination is examined. A list of "feasible" paths (i.e. paths that can a priori accommodate the requested QoS) is then deduced using the GCAC function. If no feasible path exists, the call is rejected by the source switch. Otherwise, the selected route is the shortest one (in terms of number of hops).

Delaunay graphs are highly connected, which allows us to build many alternate routes. As highlighted in Figure 3, it is always possible to avoid a blocking link in two ways using a two hop bypass. In our model the set of precomputed paths is built considering the default shortest-path ${ }^{8}$ and all the possible bypasses in case a link on this direct path does not have enough bandwidth, i.e., whenever $X<b$ (if $b$ is the amount of bandwidth requested in the call set-up). It should be noted that each blocking

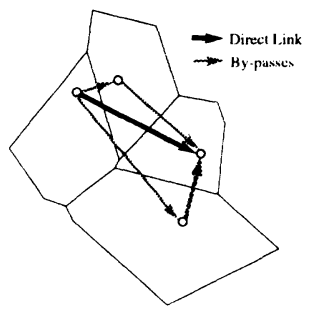

Figure 3 Alternate bypasses link introduces a penalty of one hop in the total path length ${ }^{9}$. In order to control the 'cost' of routes, the source will limit the number of penalties, rejecting the call whenever the path uses too many ressources.

\section{PERFORMANCE MEASURES}

In the following, the performance measures are first computed as conditional probabilities or averages, given a particular topology. An approximation for averaging these quantities is then discussed at the end of this section.

Passing through a spoke. Let $\mathbf{q}_{0,1}$ denote the probability of passing a spoke (in the complex node representation). The corresponding event implies that :

(i) at level-1, GCAC finds enough bandwidth on the spoke (i.e. $X^{1}>b$ );

(ii) at the physical level, it is actually possible to find a feasible route.

Let $N_{0}$ denote the number of links in the aggregation region considered and let $m_{0}=\left\lfloor\beta_{0} N_{0}\right\rfloor+1$ be the sample quantile index. Let $\Omega_{s}$ denote the event " $s$ out of $N_{0}$ links fail" (i.e. $s$ out of $N_{0}$ links have $X<b$ ). Let now $M_{0}$ denote the number of links needed to cross the peer-group (on the shortest 
path), and let $\Upsilon_{k}$ denote the event " $k$ links out of $M_{0}$ are eliminated by GCAC" (as $X<b$ ). In order to control the overhead of the paths, we impose $k \leq K_{0}$ with $K_{0}=\left\lfloor\alpha_{0} M_{0}\right\rfloor$. It is easy to see that $\mathbb{P}\left(\Upsilon_{k} \mid \Omega_{s}\right)$ is hyper-geometrically distributed,

$$
\mathbb{P}\left(\Upsilon_{k} \mid \Omega_{s}\right)=\frac{\left(\begin{array}{l}
N_{0}-s \\
M_{0}-k
\end{array}\right)\left(\begin{array}{l}
s \\
k
\end{array}\right)}{\left(\begin{array}{l}
N_{0} \\
M_{0}
\end{array}\right)}
$$

Thus, it is easy to see that :

$$
\mathbf{q}_{0,1}=\sum_{s=0}^{m_{0}-1} \sum_{k=0}^{K_{0} \wedge s} \mathbb{P}\left(\Omega_{s}\right) \mathbb{P}\left(\Upsilon_{k} \mid \Omega_{s}\right) \mathbf{q}_{0,1 \mid \Omega_{s}, \Upsilon_{k}}
$$

where $a \wedge b=\min \{a, b\}$ and $\mathbf{q}_{0,1 \mid \Omega_{s}, \Upsilon_{k}}$ denotes the probability of passing through the spoke, given $\Omega_{s}$ and $\Upsilon_{k}$. This probability describes whether a path with $k$ alternate by-passes can be found or not, i.e. wether the failing links can be by-passed. Denoting $T \in\{0,1,2,3,4\}$ as the number of links for which $X<b$ around a failing link, it is easy to see thet $T$ has a hypergeometric distribution:

$$
\mathbb{P}(T=t)=\frac{\left(\begin{array}{c}
N_{0}-s-\left(M_{0}-k\right) \\
4-t
\end{array}\right)\left(\begin{array}{c}
s-k \\
t
\end{array}\right)}{\left(\begin{array}{c}
N-M \\
4
\end{array}\right)}
$$

For $t=0$ and $t=1$, it is always possible to by-pass the failing link. For $t=2$, the probability of by-passing the failing link is $\frac{1}{2}$. This reasoning can be extended for the $k$ blocking links by introducing the set $t_{0}, \ldots, t_{k-1}$ :

$$
\mathbf{q}_{0,1 \mid \Omega_{s}, \Upsilon_{k}}=\sum_{t_{0}=0}^{2} \ldots \sum_{t_{k-1}=0}^{2} \frac{\left(\begin{array}{c}
N_{0}-M_{0}-(s-k) \\
4-t_{0} \ldots 4-t_{k-1}
\end{array}\right)\left(\begin{array}{c}
s-k \\
t_{0} \ldots t_{k-1}
\end{array}\right)}{\left(\begin{array}{c}
N_{0}-M_{0} \\
4 \ldots 4
\end{array}\right)} 2^{-\sum_{i=0}^{k-1} \mathbf{1}_{\left\{t_{i}=2\right\}}}
$$

Blocking. Let $M_{1}$ denote the number of hops needed to cross the network at level-1 (using the shortest path algorithm). The probability of passing through a link of level-1 is, by construction, $\mathbf{p}_{0,1}=\mathbf{q}_{0,1}^{2}$. Thus, it is possible to pass directly through a link of level-1 with probability $\mathbf{p}_{\mathbf{d}}=\mathbf{p}_{0,1}$. If not, two alternate by-passes could be tried instead. The probability of passing through one of the two alternate by-passes is then $\mathbf{p}_{\mathbf{a}}=\mathbf{p}_{0,1}^{2}+\left(1-\mathbf{p}_{0,1}^{2}\right) \mathbf{p}_{0,1}^{2}$ which can be restated as $\mathbf{p}_{\mathbf{a}}=\left(2-\mathbf{p}_{0,1}^{2}\right) \mathbf{p}_{0,1}^{2}$. The probability of blocking is then given by this simple expression :

$$
1-\mathcal{P}_{b}=\mathbf{p}_{0,1} \sum_{k=0}^{K_{1}}\left(\begin{array}{c}
M_{1} \\
k
\end{array}\right) \mathbf{p}_{\mathbf{d}}{ }^{M_{1}-k}\left(1-\mathbf{p}_{\mathbf{d}}\right)^{k} \mathbf{p}_{\mathbf{a}}{ }^{k}
$$

where $K_{1}=\left\lfloor\alpha_{1} N_{1}\right\rfloor$ controls the length of the path in the level-1 graph. The first term $\mathbf{p}_{0,1}$ comes from the first and the last spokes which cannot be bypassed. 
Rejection. Rejection occurs whenever the source switch is not able to find any feasible route which leads to the destination. Hence the expression,

$$
\mathcal{P}_{r}=1-\mathbf{p}_{0,1} \sum_{k=0}^{K_{1}}\left(\begin{array}{c}
M_{1} \\
k
\end{array}\right) \mathbf{p}_{1}^{M_{1}-k}\left(1-\mathbf{p}_{1}\right)^{k}\left(2-\mathbf{p}_{1}^{2}\right)^{k} \mathbf{p}_{1}^{2 k}
$$

where $\mathbf{p}_{1}$ is expressed in appendix A.2. The term $\mathbf{p}_{0,1}$ comes from the first and the last spokes GCAC which cannot be by-passed.

Crankback. The probability of crankback is defined as the probability that the call is crankbacked at least once. On a single link, the probability of crankback is then $1-\mathcal{P}_{c}^{(1)}=\mathbf{p}_{0 \mid 1}=\frac{\mathbf{p}_{0,1}}{\mathbf{p}_{1}}$. Taking by-passes into account, the expression becomes

$$
1-\mathcal{P}_{c}^{(1)}=\frac{\mathbf{p}_{\mathbf{d}} \mathbf{p}_{0 \mid 1}+\left(1-\mathbf{p}_{\mathbf{d}}\right) \mathbf{p}_{\mathbf{a}} \mathbf{p}_{0 \mid 1}^{2}}{\mathbf{p}_{\mathbf{d}}+\left(1-\mathbf{p}_{\mathbf{d}}\right) \mathbf{p}_{\mathbf{a}}}
$$

where $\mathbf{p}_{\mathbf{d}}$ and $\mathbf{p}_{\mathbf{a}}$ are defined in the previous paragraphs.

Blocking with no re-routing.. When re-routing is not allowed after a crankback, the expression of the blocking probability becomes:

$$
\mathcal{P}_{b}^{\prime}=1-\left(1-\mathcal{P}_{r}\right)\left(1-\mathcal{P}_{c}\right)
$$

Blocking without GCAC at level-1.. For comparative purposes, we have analyzed the network when the source node does not run any GCAC on the logical links. The probability of crossing a "spoke" can then be expressed as :

$$
\mathbf{q}_{0,1}^{*}=\sum_{k=0}^{K_{0}}\left(\begin{array}{c}
M_{0} \\
k
\end{array}\right) \mathbf{p}_{0}^{M_{0}-k}\left(1-\mathbf{p}_{0}\right)^{k}\left(2-\mathbf{p}_{0}^{2}\right)^{k} \mathbf{p}_{0}^{2 k}
$$

Posing $\mathbf{p}_{0,1}^{*}=\mathbf{q}_{0,1}^{*}$, the blocking probability can be expressed as :

$$
\mathcal{P}_{b}^{*}=1-\mathbf{p}_{0,1}^{*} \sum_{k=0}^{K_{1}}\left(\begin{array}{c}
M_{1} \\
k
\end{array}\right) \mathbf{p}_{0,1}^{*}{ }^{M_{1}-k}\left(1-\mathbf{p}_{0,1}^{*}\right)^{k}\left(2-\mathbf{p}_{0,1}^{*}\right)^{2} \mathbf{p}_{0,1}^{*}{ }^{2 k}
$$

Crankback without GCAC at level-1.. In this case, the crankback probability becomes :

$$
\mathcal{P}_{c}^{*}=1-\mathbf{p}_{0,1}^{*} \mathbf{p}_{0,1}^{*}{ }^{M_{1}}
$$




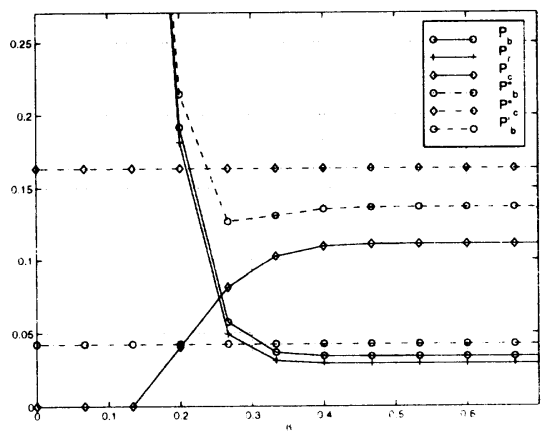

(a) Influence of $\beta$.

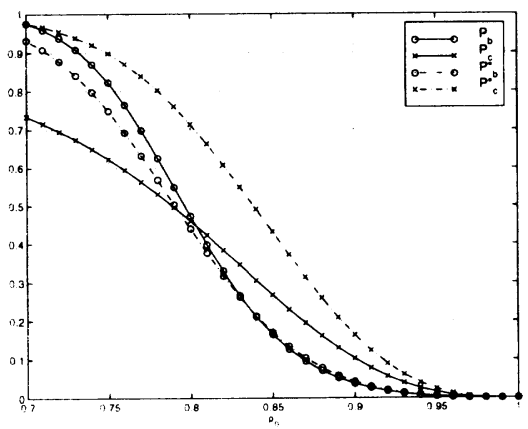

(b) Influence of $\mathbf{p}_{0}$

Averaging.. The previous quantities have to be averaged with respect to the random graphs $\pi_{0}$ and $\pi_{1}$. However the distributions of the aggregation areas and of the path lengths (in terms of hops) are not known to date. We shall assume that $M_{0}=\left\langle\frac{1}{2} \sqrt{\frac{\lambda_{0}}{\lambda_{1}}}\right\rangle$ and $N_{0}=\left\langle\frac{1}{4} \frac{\lambda_{0}}{\lambda_{1}}\right\rangle$, where $\langle$.$\rangle is the round operation$ and $\frac{\lambda_{0}}{\lambda_{1}}$ is the average number of nodes in a cell ${ }^{10}$. Furthermore, we shall assume $M_{1}=\left\langle\frac{2}{\pi} \sqrt{\lambda_{1}}\right\rangle$. where $\frac{2}{\pi}$ is the diameter of the network. Those assumptions are discussed in appendix A.1.

\section{NUMERICAL RESULTS AND ANALYSIS}

In Figure 4(a), the network behavior is represented as a function of the "aggressiveness" of the aggregation strategy for $\lambda_{0}=2000$ (2000 nodes in average) and $\lambda_{1}=33$ (33 peer-groups in average), $\mathbf{p}_{0}=0.9, K_{1}=1$ and $K_{0}=2$. We observe that, for conservative strategies (for small values of $\beta$ ) the model presents an high blocking rate caused by a high source rejection probability. Increasing $\beta$, the rejection and the blocking rates $\left(\mathcal{P}_{r}\right.$ and $\left.\mathcal{P}_{b}\right)$ decrease but the crankback rate increases (leading ot a larger routing overhead).

As expected, $\mathcal{P}_{b}^{\prime}$ is largely outperformed by $\mathcal{P}_{b}$ for any value of $\beta$ unlighting the usefullness of rerouting the calls after crankback. we note that $\mathcal{P}_{b}^{\prime}$ has a minimum for $\beta \approx 0.25$, where an optimal trade-off between rejection at the source and set-up failures(due to aggregation inaccuracy) is found.

The blocking and crankback rates when GCAC is disabled at the higher level can also be investigated. In Figure 4(a), it can be seen that this routing strategy is slightly less efficient for aggressive values of $\beta$. Futhermore, a higher crankback rate is induced by this scheme. We can conclude that performing 
GCAC (even on rough aggregated information) can help to preserve the network resources while decreasing the routing overhead.

Given the aggressiveness of the aggregation algorithm, it is also interesting to observe the network behavior as a function of the required QoS. In Figure 4(b) the probabilities of blocking and crankback for the two routing strategies are presented as functions of $\mathbf{p}_{0}$. We have chosen the same parameters of the previous plot and set $\beta$ to 0.35 . As in the previous case, performing GCAC at level-1 allows a lower crankback rate for every $\mathbf{p}_{0}$ and, then, a better network utilization. We observe that for high required bandwidths, i.e., for low values of $\mathbf{p}_{0}$, using GCAC gives worse blocking rates than the second strategy. This can be explained by the fact that performing GCAC on aggregated information can exclude logical links, with insufficient declared bandwidth, that could have accepted the call. Instead, the second strategy, not using aggregated information, avoids only the logical links that have actually caused a crankback.

\section{CONCLUSIONS AND WORK IN PROGRESS}

We obtained analytical expressions for the crankback and blocking probability as a function of the routing aggregation policy. It turned out that :

- aggressive aggregation policies perform better with respect to the blocking probability ${ }^{11}$ because of call rejection at the source;

- the GCAC function helps in selecting appropriate paths, even with very rough routing information $(\beta \rightarrow 1)$, the crankack probability is significantly reduced (w.r.t. the case where GCAC is disabled);

- however, $G C A C$ performs poorly for calls requesting a large amount of bandwidth, it could be better not to use GCAC for these calls.

Work is still going on in order to incorporate other phenomena (such as stale routing information caused by the routing update policy) in the model and for averaging more precisely the performance measures.

\section{Appendix}

\section{A.1 RANDOM GEOMETRY}

This appendix is devoted to the presentation of the Random Geometry concepts on which our analysis is based. For a more formal and exhaustive presentation of this concepts, see $[5 ; 2 ; 3]$.

In this section, $\pi$ denotes a stationary Poisson process in $\mathbb{R}^{2}$ of intensity $\lambda$. For all $u \in \mathbb{R}^{2},\|u\|$ denotes the Euclidean norm of $u$.

Voronoi Cells.. The Voronoi cell with centroid $u \in \pi$, denoted by $C_{u}(\pi)$, is the region of the plane whose points are closer to $u$ than to any other point of 
$\pi$. A Poisson process can then be used to randomly partition the plane $\mathbb{R}^{2}$. The resulting mosaic is called a Poisson-Voronoi Tessellation.

Delaunay Graph.. Two points $u, v \in \pi$ are directly connected in the Delaunay Graph, if and only if their Voronoi cells $C_{u}(\pi)$ and $C_{v}(\pi)$ have a common edge (i.e. the cells are adjacent). Such a graph and its underlying Poisson-Voronoi tessellation are represented in Figure 2(a).

Path "lengths" in the Delaunay graph.. In this paper, we are interested in the number of hops used in a path. Let us denote this measure by $\nu(\operatorname{path}(s, t, \pi))$ for two arbitrary points $s$ and $t$. For a specific paths (the "markov paths" in [3]), this measure is known explicitely as : $\nu(\operatorname{path}(s, t, \pi))=\operatorname{frac} 4 \pi \sqrt{\lambda}\|s-t\|$. More generally, scaling argument show that, for for sub-addtive routing algorithms (see [3]), $\mathbb{E}[\nu(\operatorname{path}(s, t, \pi))] \sim \kappa \sqrt{\lambda}\|s-t\|$ when $\|s-t\|$ is large, where $\kappa$ is a constant characterizing the routing algorithm. In this paper we shall pose $\kappa=1$ for simplicity.

\section{A.2 ORDER STATISTICS}

Let $\left\{X_{i}\right\}_{i \in \mathcal{I}}$ be a set of random variables. Let then define $X_{(1)}<X_{(2)}<$ $\ldots<X_{(i)}<\ldots X_{(N)}$, where $N=|\mathcal{I}|$. Given $\beta \in[0,1[$ and $m=\lfloor\beta N\rfloor+1$, the random variable $X_{(m)}$ is called the $\beta$ sample quantile.

Let us denote $Q_{b}=\mathbb{P}\left(X_{(m)}>b\right), P_{b}=\mathbb{P}(X>b)$ and $\Omega_{s}=\left\{X_{(s)} \leq\right.$ $\left.b<X_{(s+1)}\right\}$ for any $s<m$. It is easy to see that $Q_{b}=\sum_{s=0}^{m-1} \mathbb{P}\left(\Omega_{s}\right)$. Furthermore, assuming that $\left\{X_{i}\right\}_{i \in \mathcal{I}}$ are i.i.d., we get :

$$
\mathbb{P}\left(\Omega_{s}\right)=\left(\begin{array}{c}
N \\
s
\end{array}\right) P_{b}^{N-s}\left(1-P_{b}\right)^{s}
$$

\section{Notes}

1. More precisely, the Peer Group is seen as a symmetric star. All the links of the star have the same attributes and metrics.

2. Source switch: the switch which is directly connected to the host originating the call.

3. Except that no "exception-bypasses" are supported.

4. The limit of this model is that it does not consider any topological information (as explained in the following). Any real aggregation strategy should rely on the Peer-Group internal topology to compute aggregated values.

5. This choice implies that $\beta$ is the fraction of aggregated links whose bandwidth is less than $Y_{u, v}$ in the area of interest.

6. The independence relation is false, as the numbers of links aggregated in adjacent areas are correlated.

7. The parameter $b$ can represent either a peak. average or effective bandwidth, depending on the admission policy.

8. We consider the number of hops as length of a path: we shall assume for this algorithm $\kappa=1$ (see also [3]). 
9. It is a worst case assumption, as an alternate path with the same number of hops could be found as the graph is highly connected.

10. In computing the average value of $N_{0}$ two terms appear: the former is $\frac{3}{2}$, it represents the ratio between the intensities of the link and node processes at physical level (see [5]), the latter is the term $\frac{1}{6}$ and represents the fraction of the cell aggregated in a "spoke".

11. through conservative policies are preferable in order to limit crankbacks

\section{References}

[1] The ATM Forum ${ }^{1}$. Private Network-Network Interface Specification version 1. document: af-pnni-0055.000. March 1996.

[2] F.Baccelli, S.Zuyev. Poisson-Voronoi Spanning Trees with applications to the Optimization of Communication Networks. INRIA Research Report No. 3040. Nov.1996.

[3] F.Baccelli, K.Tchoumatchenko, S.Zuyev. Markov Paths on the Poisson-Delaunay Graph. INRIA Research Report No. 3420. Mai.1998.

[4] H.A.David.Order Statistics. Wiley Interscience, Second Edition, 1981.

[5] A.Frey, V.Schmidt, Marked Point Processes in the Plane - Part I. A survey with applications to Spatial Modeling of Communication Networks. Adv. Perf. Anal. 1, pp. 65-110, 1998.

[6] R.Guérin, A.Orda. QoS-based Routing in Networks with Inaccurate Information: theory and Algorithms. Proc. IEEE Infocom 97, 1997.

[7] F.Hao, E.W.Zegura, S.Bhatt. Performance of the PNNI Protocol in Large Networks. Proc. IEEE ATM Workshop 98, 1998.

[8] A.Iwata, R.Izmailov et al. QoS Aggregation Algorithms in Hierarchical ATM Networks. Proc. of IEEE ICC'98, Atlanta, June 1998.

[9] A.Iwata, R.lzmailov et al. Routing Algorithms for ATM Networks with multiple QoS requirements. Proc. IFIP WATM'95. Paris, Dec. 1995.

[10] L.Kleinrock, F.Kamoun. Hierarchical Routing for Large Networks. Computer Networks, vol.1. 1977.

[11] W.C.Lee. Topology Aggregation for Hierarchical Routing in ATM Networks. ACM Computer Communication Review, vol.25, April 1995.

[12] P.Van Mieghem. Estimation of an Optimal PNNI Topology. Proc. IEEE ATM'97, Lisboa. 1997.

[13] P.Van Mieghem. Routing in a Hierarchical Structure. Proc. IEEE ICATM'98, Colmar. 1998.

[14] J.L.Rougier, D.Kofman. A.Gravey. Optimization of Hierarchical Routing Protocols. Proc. IFIP ATM'98, Ilkley (UK). July 1998.

[15] J.L.Rougier, A.Ragozini, A.Gravey, D.Kofman. Estimation of Crankback Probability in Hierarchical PNNI network. Proc. IEEE ICATM'99, Colmar (France). June 1999.

[16] A.Shaikh, J.Rexford, K.G.Shin. Efficient Precomputation of Quality-of-Service Routes. Proc. IEEE NOSSDAV 98, July 1998.

[17] A.Shaikh, J.Rexford, K.G.Shin. Evaluation of Overheads of Source Directed Quality of Service Routing. Proc. IEEE ICNP98, October 1998. 